\title{
Iwona Bartoszewicz, Joanna SzCZEK, Artur TwOREK (Hg.): Grenzen der Sprache - \\ Grenzen der Sprachwissenschaft $I$. \\ Wrocław, Dresden: ATUT, Neisse Verlag, 2017: 290 S.
}

Der vorliegende Sammelband Grenzen der Sprache - Grenzen der Sprachwissenschaft I entstand als Ergebnis der internationalen Tagung Linguistische Treffen in Wroctaw, die seit 2006 alle zwei Jahre am Institut für Germanistik der Universität Wrocław veranstaltet wird. Die Konferenz, deren Rahmenthema den Grenzen von Sprache und Linguistik gewidmet war, fand vom 14. bis zum 16. September 2017 in Wrocław statt.

Der Band hat eine klare Struktur: Im Vorwort stellen sich die Herausgeber die Frage, wo die Grenzen der Linguistik liegen. Die Antwort auf diese Frage versuchen die Autoren des Bandes in ihren jeweiligen Beiträgen zu geben. Die Sammelmonographie umfasst 24 Beiträge, die im Folgenden kurz charakterisiert werden.

Der erste Beitrag $\mathrm{Zu}$ den Grenzen der grammatischen (Re)Analyse von Piotr Bartelik behandelt die grammatische (Re-)Analyse der Struktur mit dem Verb mieć an zahlreichen Beispielen. Der Autor präsentiert die mögliche Nutzung der Struktur unter Berücksichtigung von Transitivität, Aspekt und anderen grammatischen Kategorien, um deren Grenze $\mathrm{zu}$ bestimmen.

Der zweite Beitrag von Olena Byelozyorova widmet sich einem Phänomen der indirekten Kommunikation - dem Tabu. In der Studie wird eine Euphemisierungsstrategie präsentiert, mit der immer neue Möglichkeiten gefunden werden, Tabus im alltäglichen Sprachgebrauch zu vermeiden.

Irina Chernenok und Elena Gordeeva beschäftigen sich in ihrem Beitrag mit den Möglichkeiten und Grenzen des Übersetzens im Bereich 
der Fachsprachen. Die Autorinnen untersuchen kognitive Faktoren, die die Bedeutungsbildung im Übersetzungsprozess beeinflussen.

Federico Collaoni widmet sich in seiner Arbeit dem Thema der Grenzen der Sprache und Sprachwissenschaft in der Ökolinguistik. Der Beitrag ist in zwei Teile gegliedert: Zum einen präsentiert der Verfasser eine metalinguistische Terminologie und zum anderen zeigt er diese Disziplin und ihre Rolle in der Linguistik auf.

Anna Dargiewicz belegt am Beispiel der Online-Ausgabe der Wochenzeitung DIE ZEIT, dass Wörter keine Grenzen haben. Die Forschung basiert auf den Komposita in Presseartikelüberschriften. Der Beitrag beschreibt, wie die Autoren der Pressemitteilungen die Aufmerksamkeit der Leser auf eine sehr kreative Weise zu gewinnen versuchen.

Justyna Dolińska beschreibt in ihrem Beitrag die lokalen Modifikationen der Verben und analysiert zu diesem Zweck Partikelverben, die sich mit den lokalen Partikeln verbinden und die Struktur der Verben verändern.

Krzysztof Huszcza beschäftigt sich in seinem Beitrag mit der überregionalen Entwicklung der niederösterreichischen Zeitschrift Podium. Der Autor weist auf die Grenzenlosigkeit der Literatur im Lichte der gesellschaftlichen und historisch-politischen Veränderungen hin.

Mariusz Jakosz präsentiert in seinem Beitrag Ironie als Ausdrucksmittel des Bewertens im deutschen Online-Diskurs. Sie wird als ein sprachliches Phänomen und eine kommunikative Strategie dargestellt, die die Evaluierung der Äußerungen in den deutschen Online-Artikeln beeinflusst.

Berit Jany weist auf den Mangel an kommunikativer Fähigkeit und pragmatischem Transfer in DaF-Anfängerkursen am Beispiel der elektronischen Fremdsprachenportfolios hin.

Elizaveta Kotrova untersucht, ob die Pragmatik in den Grenzen der Sprachwissenschaft liegt. Die Autorin erklärt drei Möglichkeiten ihrer Interpretation.

Piotr Krycki erklärt mögliche Grenzen der heutigen Kommunikation. Diese ist mit verschiedenen sozialen Systemen verbunden, die oft unterschiedliche Beschränkungen aufweisen, die Störungen der Kommunikation verursachen können.

Jolanta Mazurkiewicz-Sokołowska beschäftigt sich in ihrem Beitrag mit der Bestimmung der möglichen Grenzen der individuell-subjektiven Bedeutungsanteile. Die Autorin zeigt die Methoden der Aufdeckung und Bestimmung der Bedeutungsanteile durch die Introspektion und das heterophänomenologische Verfahren.

Attila Mészáros präsentiert am Beispiel der deutschen und der slowakischen Flüchtlingsdebatte die Möglichkeiten einer vergleichenden 
linguistischen Diskursanalyse. Im Beitrag erfahren wir, mit welchen Instrumenten der linguistischen Analyse der Diskurs erfolgreich analysiert werden kann.

Renata Nadobnik definiert die Rolle der illustrativen Elemente von deutsch-polnischen Sprachführern bei der Kommunikation in typischen Alltagssituationen. Die Studie präsentiert nicht nur die Antwort auf die Zielsetzung dieser Textsorte, sondern bietet zugleich einen diachronischen Überblick über deutsche und polnische Sprachführer.

Gabriela Nitka analysiert die Funktionen des demonstrativen Determinativs taki sam (dt. der gleiche) am Beispiel der Texte aus dem polnischen Zivilgesetzbuch und dem Gesetzbuch der Handelsgesellschaften. Im Mittelpunkt des Beitrags stehen die Art der Referenzbezüge dieses demonstrativen Determinativs bei der Entwicklung der informationsstrukturellen Zusammenhänge in den Vorschriften und die Funktionen derartiger Bezüge auf der Ebene des Fachtextes.

Roman Opiłowski erforscht die Medienlinguistik und thematisiert die Funktionen des Web 3.0. Im Beitrag werden neue Forschungsfelder der Medienlinguistik im Bereich des Webs 3.0 genannt.

Paweł Rybszleger analysiert die Selbstdarstellung auf Twitter als Bestandteil einer Online-Identität und Kommunikationshandlung. Die Grundlagen der Forschung sind multimodale Elemente, die online zugänglich sind.

Monika Schönherr beschäftigt sich mit den Meinungsbekundungen in den akademischen Texten der polnischen Studierenden. Die Analyse basiert auf den Abschlussarbeiten polnischer Studierender. Die Verfasserin präsentiert die Möglichkeiten der Optimierungsstrategien, um Schwierigkeiten und Defizite zu vermeiden.

Janusz Stopyra untersucht die Grenzen der Produktivität von Bildungsmustern. Der Beitrag ist auf negativ formulierte Restriktionen fokussiert, die neben den positiv formulierten Wortbildungsregeln erscheinen und die Lehrprozesse erschweren.

Beziehungen aus dem Bereich „Arbeit und Soziales“ in ausgewählten Pressezitaten thematisiert in ihrem Beitrag Grażyna Strzelecka. Der dargestellte Wortschatz stammt aus den Jahren 1885-1886 und wird in verschiedenen Kontexten interpretiert.

Hanna Stypa beschäftigt sich in ihrem Beitrag mit einem sehr aktuellen Thema, nämlich mit dem Wortschatz der Computerspieler in der Chat-Kommunikation. Im Beitrag wird die Analyse des Jargons von Computerspielern dargestellt.

Markéta Valíčková untersucht deutsch-tschechische Übersetzungsäquivalente des Verbs lassen. Sie fokussiert auf die Idiomatik und die Methodik der Korpusanalyse. 
Rezensionen/Recenzje

Jūlija Zālìte analysiert Beteiligungsrollen und deren Einfluss auf den Sprecherwechsel in deutschen und lettischen politischen Fernsehtalkshows. Im Korpus befinden sich Auszüge von Diskussionen aus zwei deutschen und lettischen Fernsehtalkshows, die unterschiedlich unterteilt und analysiert werden.

Tadeusz Zuchewicz beschreibt in seinem Beitrag die Probleme beim richtigen Schreiben von Studierenden der Germanistik und sucht nach möglichen Lösungen.

Der Sammelband Grenzen der Sprache - Grenzen der Sprachwissenschaft ist ein interessantes und vielfaltiges Werk, das nicht nur den Sprachwissenschaftlern zu empfehlen ist, sondern auch allen, die an Linguistik und Kommunikation interessiert sind. Die Beiträge liefern aktuelle Informationen aus vielen Bereichen der Sprachwissenschaft und weisen auf die andauernde Entwicklung dieser Disziplin hin. Die Autorinnen und Autoren überprüfen, ob sich die Grenzen der Sprache verschieben und zeigen dabei darauf, dass sich die Reichweite der Forschung im Rahmen der Sprachwissenschaft ständig erweitert.

Alicja Janiszewska

(D) https://orcid.org/0000-0002-6733-4748

Universität Wrocław 